tensor and $i i)$ the expression for the heat flux in terms of the superadiabatic gradient. The contributions of the turbulent convective motions to the mean momentum and energy equation are treated consistently, and assunptions about the turbulent viscosity and heat transport are replaced by assumptions about the turbulent flow itself. The free parameters in our formalism are the relative cell sizes and their dependence on depth and latitude.

\title{
THERMAL AND CONTINUUM DRIVEN CONVECTION IN B-STARS
}

George Driver Nelson

CODE CB, Johnson Space Center

Houston, Texas, 77058

Abstract

Two regions of convective instability are present in the photosphere of a typical B-star $\left(\mathrm{T}_{\text {eff }}=30,000 \mathrm{~K} \log \mathrm{g}=4.0\right)$. One is the usual thermal instability caused by the helium ionization. The other is driven by the continuum radiation pressure in a thermally stable layer. Mixing length and anelastic modal representations of these unstable regions show that the rapid radiative cooling of temperature fluctuations limits the velocities to an amplitude of a few meters per second, much too small to account for the observed line broadening and asymetries. 